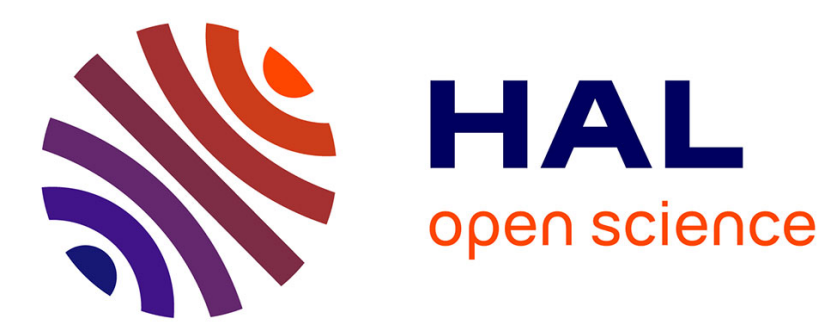

\title{
Vieux enfants, nouveaux infanticides
}

Léandro de Lajonquière

\section{To cite this version:}

Léandro de Lajonquière. Vieux enfants, nouveaux infanticides. Cliopsy, 2009, No 1 (1), p. 95-100. 10.3917/cliop.001.0095 . hal-03006302

HAL Id: hal-03006302

https://hal-univ-paris8.archives-ouvertes.fr/hal-03006302

Submitted on 15 Nov 2020

HAL is a multi-disciplinary open access archive for the deposit and dissemination of scientific research documents, whether they are published or not. The documents may come from teaching and research institutions in France or abroad, or from public or private research centers.
L'archive ouverte pluridisciplinaire HAL, est destinée au dépôt et à la diffusion de documents scientifiques de niveau recherche, publiés ou non, émanant des établissements d'enseignement et de recherche français ou étrangers, des laboratoires publics ou privés. 


\title{
Vieux enfants, nouveaux infanticides
}

\author{
Leandro de Lajonquière \\ Traduction et adaptation avec l'auteur : \\ Ilaria Pirone et Laurence Gavarini
}

Ces dernières années, une série d'idées singulières a surgi au sujet de I'enfance. Il est dit par exemple que les enfants d'aujourd'hui sont plus intelligents et plus rapides que ceux d'autrefois, qu'ils savent ce qu'ils veulent et ont des opinions, au point que nous doutons qu'il s'agisse encore d'enfants ou, en d'autres termes, que leur manière d'être continue de correspondre à ce qu'on nommait enfance par le passé. Ces changements présupposés de l'enfance font que nous nous sentons obligés $d^{\prime}$ « adapter » et de « moderniser » notre façon de les accueillir au monde - notre façon de les éduquer, de leur adresser la parole -, puisque le savoir sur l'enfance que nous pouvons avoir, ayant été enfant nous-mêmes, ne peut qu'être imprécis, ne peut pas être utilisé comme critère et doit être mis de côte en tant que « chose du passé ».

On donne pour acquis qu'actuellement « nous aimons nos enfants » et que la meilleure preuve en est que nous leur demandons leur opinion et que nous les stimulons à participer à différentes discussions et à des activités variées au quotidien. Dans cette même veine, nous sommes aussi toujours prêts à répertorier toute sorte de maltraitance envers les enfants, et d'ailleurs, par la loi, nous reconnaissons pour ces « enfants différents d'aujourd'hui » une série de droits ${ }^{1}$ !

On pense que l'enfance pourrait bien être en voie de disparition ou possiblement qu'elle aurait une durée plus courte pour les « enfants si différents d'aujourd'hui » (Postman, 1982 ; Ariès, 1973). Il y a aussi le fait que les adultes, plus conscients et éclairés que jadis, doivent tolérer des habitudes, des intérêts et des goûts singuliers et très nouveaux. En somme, on croit que nous voyageons dans le temps, en suivant une échelle d'évolution qui irait de la tolérance médiévale à l'infanticide, jusqu'à la reconnaissance légale de ce qu'on appelle l'intérêt de l'Enfant.

Évidemment, je suis persuadé que les temps changent. Néanmoins, je doute que la vie au contact des enfants évolue dans une direction aussi claire et distincte tel qu'on le juge aujourd'hui. Qui plus est, $j$ 'estime que le fait de ne pas mettre en doute la certitude d'une telle ligne d'évolution est un indicateur que quelque chose ne va pas bien dans le cours de notre vie quotidienne.

Si je me questionne à propos de l'évolution de nos habitudes et coutumes, cela ne signifie pas penser que la ligne de l'histoire suivrait - à l'inverse - un mouvement de dégradation. Tout simplement je crois que la famille, la tradition et la propriété, parmi d'autres productions humaines, sont prises dans l'air du temps et donc se présentent dans la société selon le dernier cri de la mode.

On ne peut donc pas parler d'évolution ou de dégradation, pour ces dernières, mais simplement de changements historiques (Lajonquière, 2000), qui nous disent quelque chose de la façon dont nous nous rêvons comme toujours autres que nous-mêmes et, par conséquent, de la façon dont nous rêvons aussi le lien de constante haïnamoration, avec ces êtres
1. Le Brésil reconnaît aux mineurs de douze ans le droit de jouer et le droit de réfuter les critères d'évaluation scolaire. Cf. Principe Septième de la Déclaration des Droits de I'Enfant et Art. 53 du Statut de l'Enfant et de L'Adolescent. Loi 8069 du 13/07/1990. 
tous petits appelés enfants, produits de ce malentendu qui habite la vie sexuelle dite adulte et qui insiste à « faire des familles ».

Ceci ne veut pas dire non plus que ces changements n'ont pas d'importance. Si le temps actuel peut se caractériser par quelque chose, c'est justement par le fait que nous pouvons nous soutenir de l'idée que nous sommes capables de promulguer des lois pour garantir, par exemple jusqu'au droit des enfants à jouer.

Jusqu'à quel point - tranquilles d'esprit - réfutons-nous la possibilité que l'infanticide soit encore présent parmi nous ? Les enfants d'aujourd'hui ne meurent pas en masse asphyxiés par «négligence » comme les bébés prémodernes dans le lit parental, mais ceci n'empêche pas que nous retrouvions dans les pages des faits divers des quotidiens, d'innombrables manières, anciennes et nouvelles, de les tuer.

Je fais référence ici à un infanticide, que l'on pourrait peut-être qualifier de symbolique. De nos jours, d'une certaine façon, le monde des vieux pêche par omission vis-à-vis des petits, actuellement «abandonnés » spirituellement. Et cette omission est - évidement - aussi « concrète ». Il n'est pas rare que l'enfant finisse "abandonné", oublié, de fait par la machine bureaucratique et judiciaire qui prétend protéger les droits "de L'Enfant". Dans le même ordre d'idées, la profusion de contrôles exercés par des agents spécialisés dans la protection de l'enfance et de la famille ne sont plus que des initiatives disciplinaires, ce que Laurence Gavarini et Françoise Petitot (1998) ont appelé "la police des fantasmes".

L'insistance actuelle à propos de la supposée bonté démocratique et de l'amour des adultes, est source d'une innombrable quantité de droits pour L'Enfant, mais n'est pas synonyme de la nécessaire implication dans la vie des adultes avec ces petits êtres venus au monde après nous. Elle indique plutôt notre refus de maintenir ouverte la question qui se pose toujours : comment être sûr de quelque chose et en parler aux enfants ?

Une telle interrogation révèle l'autre face de l'impossibilité d'établir un rapport entre petits et vieux, un rapport clair entre les générations. De cette impossibilité, on ne veut rien savoir, et on n'en veut rien savoir d'une façon que je définirais de cynique, masquée par une préoccupation exacerbée pour des questions telles que l'égalité de genres et par des divagations psychopédagogiques sur la relation adulte-enfant.

Quand un enfant arrive dans les bras de sa mère, bien que ce moment ne marque le début de rien, car l'histoire de l'enfant était déjà en cours, une différence s'installe entre un avant et un après. À ce moment-là, la femme est confrontée au fait d'accepter, ou non, de devenir mère de ce petit qui vient au monde toujours plus ou moins étranger par rapport à ceux qui habitent déjà ce monde. En effet les bébés dorment pendant la journée et sont plus sociables la nuit, ils pleurent pour des choses que les adultes ne comprennent pas, font des grimaces, parlent une langue qui n'est pas facile à comprendre. Ces exemples, ce sont des exemples parmi d'autres de ce que les adultes perçoivent au quotidien comme des bizarreries des enfants. Le fait de recevoir un enfant comme s'il était un étranger ne signifie pas le percevoir insolite de la même façon qu'un extraterrestre ou qu'un sauvage. L'arrivée du nouveau-né dans le monde adulte est un événement familial qui revêt une inquiétante étrangeté par le fait qu'il ravive un état infantile supposé dépassé par l'adulte concerné. Freud (1919) parle de l'inquiétante étrangeté quand le sujet assiste au retour inespéré de quelque chose qui a été autrefois familier.

Parfois, au lieu d'un accueil du nouveau-né dans I'hospitalité, rendant possible un processus de «familiarisation », il se peut que la naissance 
produise, au contraire, un bébé ayant figure d'extraterrestre ou de sauvage. Le caractère inquiétant de l'étrangeté est à ce point exagéré qu'il devient une étrangeté impossible à être reconnue.

Celui qui se prend pour civilisé prétend maintenir une certaine distance visà-vis de l'individu qu'il considère comme sauvage ${ }^{2}$. D'un autre côté on ne veut rien savoir de l'extraterrestre sinon qu'on veut continuer à garder nos distances, une attitude qui nous permet en même temps de l'adorer, d'en rêver mais aussi de le fuir au cas où il s'approcherait un peu trop. Finalement tant l'extraterrestre que le sauvage sont traités différemment d'un étranger à qui on suppose - avec plus ou moins de sympathie - de posséder des choses d'un monde Autre $^{3}$ qu'il pourra nous raconter.

Une mère parle à son bébé dans l'attente qu'il apprenne sa langue (maternelle) et qu'ainsi il puisse lui raconter ces choses d'un Autre monde, pour que tous deux, la mère et son enfant, parviennent à être moins étrangers l'un à l'autre et, par conséquent, plus familiers. Une mère suppose au petit nouveau-né la même initiative énonciative que la sienne, ainsi qu'une même capacité de dialogue.

Une femme projetée dans le désir de Femme peut devoir faire face à un homme dans sa vie. Comme preuve du malentendu du commerce entre les sexes, surgit un bébé, qui réintroduit la différence irréductible entre la Femme et la mère. Le petit être ouvre à nouveau la cause du désir et ainsi vient faire signe de l'absence de rapport sexuel qui habite le monde des adultes. Une mère peut franchir ou non le pas de donner le fruit de cette non-rencontre du rapport sexuel à l'homme - élu pour inventer la place du père et pour lequel elle se met en place d'être l'objet cause de son désir d'homme.

De fait, les femmes/mères précipitent les hommes dans I'aventure d'être père. Ainsi, une femme métaphorise du père, c'est-à-dire vit l'expérience de la production d'une inversion subjective ${ }^{4}$ de la position infantile de demander au père la donation d'un objet impossible - un bébé en tant que suture du narcissisme. Et plus encore, la métaphore creuse un écho dans le registre du sens produisant ainsi un évidement du savoir sur l'être père.

L'arrivée d'un petit être implique un réaménagement du monde, puisqu'une différence. Cette différence contribuera avec l'action du temps au "devenir adulte". Tout adulte, indépendamment de ce que cet adulte soit ou non une femme convertie en mère, quand il s'adresse à un enfant lui demande d'abandonner la condition étrangère de l'infans. Il n'y a pas de vestiges historiques qui ne montrent que les cultures ont toujours mis les enfants dans une certaine forme de quarantaine par rapport au monde adulte et,de la même manière, que les adultes ont toujours symbolisé pour eux-mêmes et pour les enfants la différence réelle, le manque de rapport entre les uns et les autres.

Pendant qu'il laisse du temps au temps, l'adulte éduque un enfant en pariant sur le dépliement de la différence posée dès l'origine par la simple arrivée de l'infans. La disponibilité métaphorique de l'adulte génère un temps destiné à être donné comme temps d'attente au petit nouvellement arrivé. L'enfant pris dans ce dispositif temporel $n$ 'a toujours pas accès au point de vue de l'adulte - le désir, en cause dans la demande éducative. Pour cette raison, il commence à supposer - et à désirer - chez l'adulte un savoir vivre.

Néanmoins quand arrive son tour, l'enfant de jadis - maintenant adulte découvre que le point de vue qu'il supposait aux adultes à l'aube de sa vie n'était pas aussi savant qu'il l'imaginait et que le temps - cette attente imposée par sa mise en quarantaine - était finalement un temps qu'il fallait vivre, un temps à consommer au delà de toute maîtrise éducative supposée.
2. Si le soi-disant civilisé le considère comme un bon sauvage, alors il voudra l'étudier de façon minutieuse et scientifique pour, ainsi, savoir en mesurer exactement la différence et pouvoir de cette manière, effacer l'étrange mystère qui anime autant I'un, qu'il angoisse I'autre. Au contraire s'il s'agit d'un mauvais sauvage, le civilisé tentera de se débarrasser de leur bizarrerie hasardeuses par le biais de

l'extermination.

3. La graphie permet de faire allusion à l'inconscient, nommé par la psychanalyse de tradition lacanienne avec le terme Autre.

4. À mon avis on pourrait dire que cette inversion est le résultat d'une conjonction entre une condensation et un déplacement signifiants. 
5. Énonciation qui témoigne de la castration de l'Autre.

6. Le préfixe im de impropre n'est pas ici dans un sens privatif, mais signifie quelque chose de non approprié à la consommation par le fait d'être trouble. Le nom propre est un paradoxe puisque il s'agit souvent d'une sorte de copropriété : plusieurs d'entre nous recevons un même nom. Par ailleurs le nom est sali par le désir parental en cause dans l'acte même de la nomination.
L'arrivée d'un bébé dans une histoire en cours instaure une tension dans le domaine du discours entre le côté infans et le côté adulte. Les deux termes ne sont pas des points d'une lignée génético-évolutive vers la raison, mais des positions du discours en relation au désir, à la parole Autre ${ }^{5}$.

Être adulte signifie paradoxalement ne pas-Être. Dans un certain sens, « l'adulte est » celui qui ne peut pas éviter de se confronter à l'impossibilité de parler en son nom propre, c'est-à-dire au nom im/propre ${ }^{6}$ du désir qui I'habite et qui le fait manquant. Cette position implique que, tant cet enfant qu'il a été pour les autres, ainsi que cet enfant qu'il n'a pas été, mais qui était attendu par eux, soient objet de refoulement psychique. Le sujet n'a pas connaissance de « cet être pour l'Autre » et donc ne parvient jamais à devenir l'adulte développé et normal dessiné par les diverses psychologies. Le soi-disant adulte est simplement un vieux.

Pour qu'un vieux advienne avec le temps au lieu où se trouve cette petite créature, il est donc nécessaire que cet autre, qui est là déjà vieux, prenne comme métaphore la non-rencontre inévitable avec ce petit être dans le monde. Quand l'infans cesse de l'être, - lorsqu'il sera devenu vieux l'enfance commence à exister comme déjà perdue, en inscrivant la présence d'une absence dans un monde toujours vieux. Une enfance n'existe que comme perdue, méconnue, refoulée et ainsi ne cesse de ne pas s'écrire, de ne pas s'inscrire, d'insister en « nous ». Elle insiste comme différence temporelle - énigme - et de cette manière elle nous rend étranges face au présent et nous rend étrangers face à nous-mêmes.

Quand un vieux rencontre un enfant il se reflète en lui comme si c'était dans un miroir. Il le regarde les yeux dans les yeux, espérant ainsi que de la profondeur de ce regard, lui revienne sa propre image à l'envers. Il espère finalement, ne pas se voir assujetti à la castration, et pouvoir ainsi s'inscrire à nouveau dans le temps pour profiter jusqu'à la dernière goutte de ce qui lui est resté de l'enfance « perdue »: I'infantile. Justement, l'adulte investit narcissiquement l'enfant, dans l'espoir toujours vain d'épuiser cet infantile qui n'arrête pas de ne pas revenir, pour ainsi finalement tout savoir de «son » enfance et de cette façon être un adulte vraiment pour de vrai comme disent les enfants -, et pas simplement un vieux.

Le savoir insu, dont on pense que l'enfant est le dépositaire, fait de lui un étranger chez qui on voudrait écouter les histoires d'un Autre monde. Mais cela s'avère impossible, puisque finalement nous prétendons qu'il nous fasse le récit de cet étranger qui nous habite. De ça (nous faisons référence ici à I'Inconscient), nous ne pouvons connaître qu'une partie : d'une part, par le fait que les enfants, demeurent toujours un peu inquiétants et qu'ils nous renvoient au cours de la vie le fait d'être toujours étrangers à ce que nous sommes ; d'autre part, par ce mécanisme même, nous offrons une hospitalité au ça. Malgré cela, le malentendu entre les générations, qui découle du manque de rapport, n'empêche pas le dialogue et au contraire l'alimente, en rendant ainsi possible une éducation.

Éduquer signifie transmettre des marques symboliques qui permettent au petit être de jouir de l'énonciation dans le champ de la parole et du langage à partir duquel, il lui soit possible de se lancer dans les entreprises impossibles du désir. Le déroulement d'une éducation, d'une filiation symbolique familiarisante, présuppose que l'adulte reçoive l'enfant comme $s^{\prime} i l$ était un étranger passible de devenir plus ou moins familier, mais jamais un clone.

Une éducation est en effet possible au-delà de sa propre impossibilité - elle aussi en germe dans les rêves des grands. Nous, petits d'hier, on a tous démarré grâce à « un coup de pouce » dans la traversée dans la mesure où chacun de nous a inversé la demande éducative, en creusant un espace 
pour soi dans les rêves des autres.

Les enfants arrivent à profiter d'une éducation, au delà de l'impossibilité de L'Éducation, comme Freud l'avait déjà annoncé, dans la mesure où persistent de l'infantile et de l'inquiétante étrangeté dans l'adulte déjà devenu plus ou moins familiers à d'autres adultes, dans un monde où tout ce qui est familier est un peu étrange et tout ce qui est étranger finit par nous sembler un peu familier.

Néanmoins une éducation peut ne pas avancer dans son déploiement aboutissant ainsi à une voie sans issue. Ainsi, I'enfant commence à avoir des difficultés à surmonter dans le processus de familiarisation ou de filiation. Au même moment, l'enfant cesse de s'adresser aux autres - à l'Autre - en tant qu'un « enfant avec nom et prénom » et il commence à errer avec une étiquette accrochée sur laquelle sont notés tous ses besoins ou ses handicaps plus ou moins spéciaux, ainsi que ses goûts et intérêts dignes d'un autre monde. L'enfant court alors le risque de se retrouver à la merci de la turbulence des temps sans beaucoup de poésie et de grâce.

Une éducation peut s'accomplir difficilement, quand pour un adulte il devient impossible de déplier la non-rencontre avec un enfant, ou si on préfère, de soutenir l'impossibilité de l'Éducation - au sens freudien. Cette impossibilité adulte peut donner lieu à diverses suppositions à propos de l'exceptionnalité infantile. Ainsi les petits restent à la merci du manque d'opportunité d'être différents de la façon dont ils sont supposés idéalement être. La supposition de l'exceptionnalité place l'enfant hors du lien social où le familier et l'étranger sont les côtés de la même bande de Mœbius.

L'éducation d'un sauvage et d'un extraterrestre sont a priori d'un accomplissement difficile. La contradiction est au cœur de leurs propres énoncés. Une éducation ne peut se réaliser que si, dans la non-rencontre avec un enfant, les adultes laissent émerger leur propre étrangeté à euxmêmes, qui a ses racines dans le manque de rapport sexuel. Tant le sauvage que l'extraterrestre représentent l'impossibilité d'accueillir le retour de la différence. Les deux sont le contraire de la présupposée mêmeté du nous-mêmes. Ni l'un ni l'autre ne peuvent habiter le même monde d'un individu qui pense pouvoir être identique à lui-même.

La thèse que les enfants d'aujourd'hui sont différents, plus intelligents que ce que nous étions dans l'enfance, n'est pas nouvelle. Il est curieux de constater comment au cours de l'histoire la «vieille garde » a toujours décerné les mêmes attributs aux nouveaux-nés. Un enfant d'aujourd'hui est aussi différent de nous, que nous l'avons été et continuons de l'être par rapport à nos vieux. La solidarité mœbienne entre l'étranger et le familier se renouvelle sans cesse, à moins que nous coupions la bande de Mœbius.

L'insistance aujourd'hui sur ladite différence des enfants est très grande. Cette insistance les rend « tellement, mais tellement différents » et indique une bévue dans la façon dont l'adulte adresse la parole à un enfant. La « grande, mais si grande différence d'aujourd'hui » ne fait pas - comme elle devrait le faire - de l'enfant un étranger, mais plutôt elle en fait un sauvage ou un extraterrestre.

Si les enfants semblent des sauvages ou des extraterrestres, il y a quelque chose qui dysfonctionne. Une différence ne peut que donner lieu à un « plus du même », c'est-à-dire à une autre différence qui rénove la dialectique étrange-familier. Les petits détails de la vie quotidienne actuelle en compagnie des enfants montrent au contraire une difficulté à déplier la différence, le reste inquiétant que l'arrivée d'un nouveau-né produit.

Par ailleurs nous n'aimons ni ne haïssons nos enfants, ni plus ni moins qu'autrefois. Tout simplement ils sont comme toujours des objets 
condensateurs de haïnamoration. Nous les haïssons, car ils disent toujours la vérité, non pas celle de la religion ou de la Science, mais celle refoulée du monde des vieux : le manque de rapport sexuel et la condition transitoire de l'existence. On les aime car la vie en leur compagnie nous offre aussi plus d'un alibi pour persévérer dans le fait qu'on ne veut rien savoir de ces vérités. Néanmoins si nous insistons sur la supposée pureté de notre amour c'est parce que l'amalgame haïnamoration semble procéder de I' intolérable. En coupant la bande de Mœbius, on sépare l'un et l'autre. L'amour pur fait du bébé un enfant quelconque, une sorte de «bon sauvage » ou d'enfant mort, comme celui dont parlent les pédagogies orthodoxes (Carmago, 2006). La haine évoque les images du méchant sauvage et de l'extraterrestre cultivées par des élucubrations pédagogiques et sociologiques à penchant apocalyptique.

Le fait de prétendre adapter l'éducation aux enfants « si différents d'aujourd'hui » est un mauvais présage. Il n'y a pas d'éducation possible si le petit est marqué en tant que sauvage ou extra-terrestre. Ainsi, si des enfants restent à la marge, comme $s$ 'ils étaient des sauvages ou des extraterrestres c'est parce que tout simplement on les laisse de côté quand on renonce à l'acte d'éduquer. On les laisse de côté pour ne rien savoir d'eux - pour ne pas découvrir ce qui nous rend étranges à nous-mêmes.

Le renoncement à l'acte d'éduquer (Lajonquière, 1999) est une forme d'infanticide. Renoncer à adresser en nom propre la parole à un enfant n'est pas un geste susceptible d'être recherché ou évité volontairement. Néanmoins il semble qu'aujourd'hui ce soit plus monnaie courante que ce que I'on est disposé à reconnaître.

La tendance à l'infanticide symbolique n'est pas une malédiction divine, ni même la conséquence d'un geste maléfique de quelque tyran occasionnel, mais plutôt le résultat du fait que nous refusions aux enfants le seul droit qui compte, à savoir le droit de revendiquer une éducation.

Pour que ces petits puissent revendiquer une simple place dans le monde on doit se donner le temps pour que, dans ses petits détails, notre vie quotidienne ait quelque chose d'étranger au présent, à la soi-disant réalité, aux marchés financiers et à ce qui se passe à la télévision. On doit se donner le temps pour ainsi donner le temps au temps afin que l'étranger revive le familier et vice-versa. C'est là notre devoir à moins que même les enfants d'aujourd'hui n'arrivent - à leur tour - à conquérir un peu de vieillesse.

\section{Bibliographie}

Ariès Philippe (1973). L'Enfant et la vie familiale sous l'Ancien Régime, Paris : Seuil. Camargo, ACCS. de (2006). Educar : uma questão metodológica ?, Petrópolis : Vozes. Freud S. (1919). L'inquiétant, in Oeuvres complètes, vol. XV, Paris : PUF, 2002, pp. 147-188.

Lajonquiere L. de (1999). Infância e Ilusão (Psico)Pedagógica. Petrópolis : Vozes.

Lajonquiere L. de (2000). Psicanálise, modernidade e fraternidade, in Maria Rita Kehl, A Função Fraterna, Rio de Janeiro : Dumara.

Postman Neil (1982). The Disappearance of Childhood, New-York : Delacorte Press.

Pour citer ce texte :

Lajonquière $L$. de, Vieux enfants, nouveaux

infanticides, trad. I. Pirone, L. Gavarini, Cliopsy, $\mathrm{n}^{\circ} 1$, 2009, pp. 95-100. 